\title{
Lobectomy as a Complication of Pulmonary Infarct after Sub segmental Pulmonary Embolism
}

\author{
John F Lazar*, Paulovich K and Hancock P
}

Pinnacle Health System, USA

\begin{abstract}
We present a case of a 41 year old African-American male with a history of thromboembolic events that was found to have a SSP as a result of pulmonary infarct. Patient underwent definitive surgical intervention, lobectomy, after failure of initial management with thoracostomy tube and video-assisted thoracoscopic (VATS) with wedge resection and pleurodesis.
\end{abstract}

\section{Introduction}

Pulmonary infarction as a complication of PE is a rare clinical entity. A SSP resulting from pulmonary infarction is even rarer with treatment tailored on case-by-case bases. Although initial treatment of SSP is insertion of thoracostomy tube, it can include a VATS with wedge resection and pleurodesis and in extreme cases a lobectomy.

\section{Case Presentation}

A 41 year old African-American male with recent history of PE secondary to unprovoked deep venous thrombosis presented to an outpatient surgery clinic for lipoma evaluation. The patient complained of shortness of breath on exertion and cough during previous several days and on physical exam had absent breath sounds. He was referred to the Emergency Department, where plain chest radiograph (CXR) confirmed a left pneumothorax with right mediastinal shift. A 14 French pigtail catheter was inserted with re-expansion of the left lung on CXR. A spiral CT showed emboli in the right and left lower lobe segmental pulmonary arteries, left lower lobe patchy opacities consistent with infarct and an area of cavitation adjacent to the left lower lung pleura.

During hospital admission there was a persistent expiratory air leak necessitating VATS, wedge resection and pleurodesis. Intra-operatively there was left lower lobe necrosis and adherence of the pleura to the chest wall (Figure 1). A partial decortication was performed, separating the necrotic portion of the lung from the chest wall followed by a wedge resection and talc pleurodesis. Tissue pathology revealed focal hemorrhage and necrosis consistent with lung infarct. An IVC filter was placed postoperatively. He was discharged on Coumadin after both chest tubes were removed with no evidence of pneumothorax on post-operative day 7 .

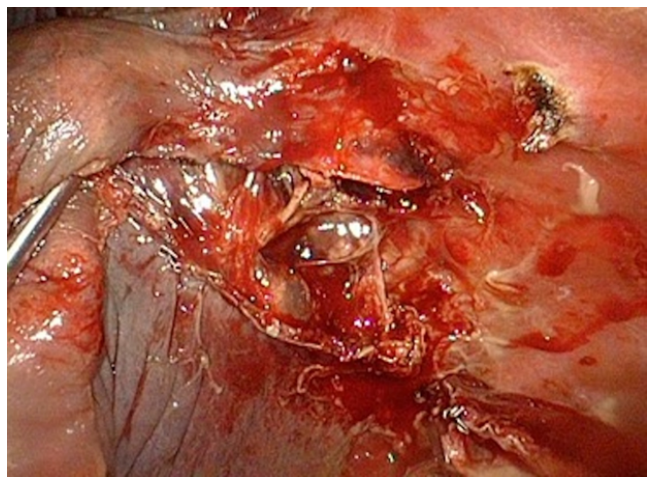

Figure 1: Devitalized left lower lobe during initial surgery
A routine CXR prior to 10 day clinic follow-up showed a recurrent pneumothorax. A pigtail thoracostomy tube was placed and CT scan showed persistent necrosis of the basilar segment of the lower lobe. He was taken back to the operating room for definitive managements with lower lobectomy, lymph node dissection and total decortication. The patient was discharged home after an unremarkable post-operative day 6 .

\section{Discussion}

Pulmonary infarction is a rare consequence of PE since collateral flow from the bronchial arteries and prolific capillary anastomoses typically compensates for the occluded pulmonary vessel and prevents ischemic tissue damage. It is seen in only $10 \%$ of patients with $\mathrm{PE}$ $[1,2]$. The incidence is higher in autopsies of patients with fatal PE [3]. A recent retrospective comparative study by Kirchner et al. evaluated possible causes for infarction with no correlation with cardiac congestion, malignancy, chronic bronchitis, clot burden, age or severity of symptoms. The main predisposing factor was peripheral location of vascular occlusion rather than large proximal occlusions [4]. This coincides with prior studies which showed the occlusion of pulmonary vessels smaller than $3 \mathrm{~mm}$ are likely beyond the physiologic anastomosis of the pulmonary and bronchial arteries and are thus predisposed to ischemia and infarction $[5,6]$. This is consistent with our patient whose area of necrosis was distal and adjacent to the pleura (Figure 2).

Pneumothorax secondary to pulmonary infarction is a rarer complication than infarction alone; the incidence of which has not been reported [1,7]. Publications regarding SSP as the result of pulmonary emboli are limited to a handful of case reports. Most cases describe septic emboli as the source of infective necrosis and cavity formation leading to rupture and pneumothorax $[8,9]$. Typically, the patient has an underlying lung condition that predisposes them to rupture. Our patient did not fit into either of these groups, which may explain his unusual clinic course.

*Corresponding author: John F Lazar, Pinnacle Health System, USA, Tel: 9177711530; E-mail: jflazar@me.com

Received May 30, 2017; Accepted June 01, 2017; Published June 07, 2017

Citation: Lazar JF, Paulovich K, Hancock P (2017) Lobectomy as a Complication of Pulmonary Infarct after Sub segmental Pulmonary Embolism. Health Care Current Reviews 5: 199. doi: 10.4172/2375-4273.1000199

Copyright: $\odot 2017$ Lazar JF, et al. This is an open-access article distributed unde the terms of the Creative Commons Attribution License, which permits unrestricted use, distribution, and reproduction in any medium, provided the original author and source are credited. 
Citation: Lazar JF, Paulovich K, Hancock P (2017) Lobectomy as a Complication of Pulmonary Infarct after Sub segmental Pulmonary Embolism. Health Care Current Reviews 5: 199. doi: 10.4172/2375-4273.1000199

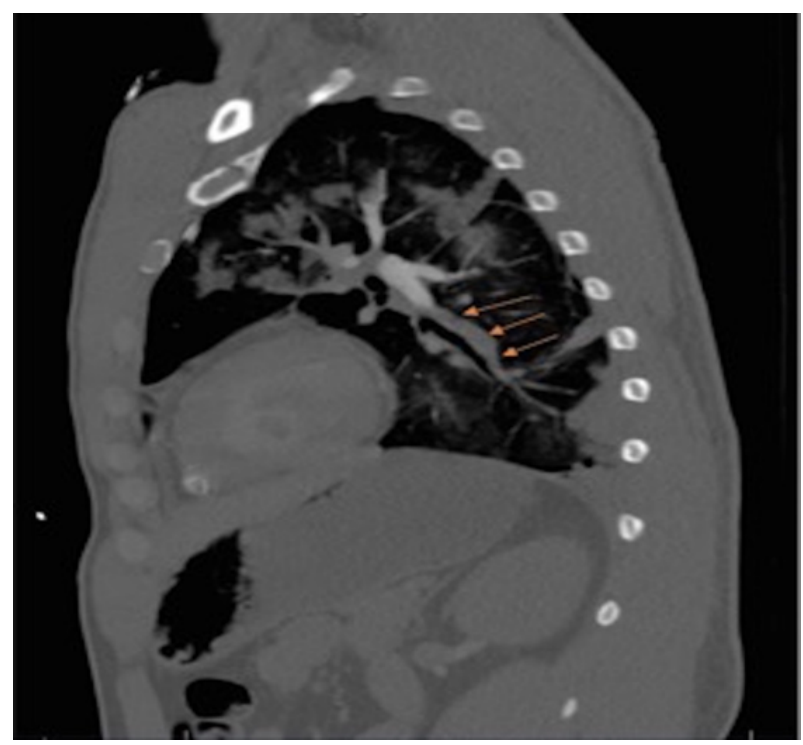

Figure 2: Axial image of CT with involved pulmonary artery branch indicated by arrows.

Many times SSP requires immediate insertion of a thoracostomy tube. An air leak is commonly seen and usually resolves without further intervention. The likelihood of resolution is low if persistent more than 2 days and necessitates surgical intervention with VATS. Several studies have shown VATS patients to have shorter hospital stays, less postoperative pain and blood loss than thoracotomy patients [10-12]. SSP recurrence rates, however, are higher in VATS than in thoracotomy (3.8\% versus $1.8 \%$ ) [13]. They did mainly report on treatment of SSP caused by bleb disease with VATS or thoracotomy. Our patient had a different pathophysiology.

Due to ongoing ischemic changes, lobectomy was required and was the appropriate surgical intervention after wedge resection failed. $\mathrm{PE}$ complicated by pulmonary infarction as a cause of SSP, albeit rare, should be included amongst the physician's considerations. When such rare diagnosis are encountered, it is imperative for clinicians to employ unconventional treatment modalities. We successfully treated SSP as a complication of pulmonary infarction with a surgical intervention, VATS and lobectomy; a novel approach to an uncommon complication hardly encountered in current literature.

\section{References}

1. Mathieson P, Reckless I, Ferrett C (2012) Secondary spontaneous pneumothorax: A rare complication of pulmonary embolism. BMJ Case Rep.

2. Vaideeswar P (1998) Cavitary pulmonary infarction - A rare cause of spontaneous pneumothorax. J Postgrad Med 44: 99-100.

3. Mordeglia F, Rios J, Dutrey D (1965) Anatomoclinical study of pulmonary embolis in patients with or without pulmonary infarction. Medicina (B Aires) 25 360-368.

4. Kirchner J, Obermann A, Stückradt S (2015) Lung infarction following pulmonary embolism: A comparative study on clinical conditions and CT findings to identify predisposing factors. Forschr Röntgenstr 187: 440-444.

5. Tsao MS, Schraufnagel D, Wang NS (1982) Pathogenesis of pulmonary infarction. AM J Med 72: 599-606.

6. Karsner HT, Ghoreyeb AA (1913) Studies in infarction: III. The circulation in experimental pulmonary embolism. J Exp Med 18: 507-511.

7. Lim K, Tan L, Liam C (2001) An unusual cause of secondary spontaneous pneumothorax in a 27 years old man. Chest 120: 1728-1731.

8. Wilson AG, Josephe AEA, Butland RJA (1986) The radiology of aseptic cavitation in pulmonary infarction. Clin Radiol 37: 31-37.

9. Lippy LS, King TE, Laforce FM, Schwarz MI (1985) Pulmonary cavitation following pulmonary infarction. Medicine 64: 342-348.

10. Schoenenberger RA, Haefeli WE, Weiss P, Ritz RF (1991) Time of invasive procedures in therapy for primary and secondary spontaneous pneumothorax. Arch Surg 126-764.

11. Vohra HA, Adamson L, Weeden DF (2008) Does video-assisted thoracoscopic pleurectomy result in better outcomes than open pleurectomy for primary spontaneous pneumothorax? Interact Cardiovasc Thorac Surg 7: 673.

12. Shaikhresai K, Thompson Al, Parkin C (2011) Video-assisted thoracoscopic surgery management of spontaneous pneumothorax-long-term results. Eur $\mathrm{J}$ Cardiothorac Surg 40: 120 .

13. Pages PB, Delpy JP, Falcoz PE, Thomas PA, Filaire M, et al. Videothoracoscopy Versus Thoracotomy for the treatment of spontaneous pneumothorax: $A$ propensity score analysis. Ann Thorac Surg 99: 258-263. 